\title{
MATERIALIZACIÓN DEL DERECHO DE ACCESO A LA JUSTICIA MEDIANTE LA CONCILIACIÓN EXTRAJUDICIAL EN DERECHO EN EL DISTRITO JUDICIAL DE SINCELEJO, COLOMBIA, DURANTE LOS AÑOS 2016 - 2017
}

\author{
Katia Marcela Palencia Sánchez ${ }^{1}$ \\ Nubia Elena Valdelamar Támara² \\ Cristian Daniel Pérez Avendaño ${ }^{3}$
}

\section{Resumen}

La conciliación extrajudicial en derecho, celebrada en el Centro de Conciliación constituye una herramienta para garantizar el efectivo acceso a la administración de justicia, en la presente investigación se describió y conceptualizo la conciliación extrajudicial como derecho fundamental, su historia, existencia y competencia de los centros en el distrito judicial de Sincelejo. Se desarrolló con base a un diseño metodológico organizado en distintas fases, principalmente un análisis de las estadísticas del Centro de Conciliación de la Corporación Universitaria del Caribe CECAR, durante los años 2016 y 2017, el cual permitió la realización de los resultados y conclusiones, identificando el que el aporte del Centro de Conciliación de CECAR

1 Doctorante en Ciencias políticas y de la Administración y Relaciones Internacionales, Magister en gobierno y Administración Pública, Especialista en Derecho Público. Abogada. Adscrita al Centro de investigación Sociojurídicas de la Corporación Universitaria del Caribe GISCER. Directora de Consultorio Jurídico y Centro de Conciliación de CECAR. Email: Katia.palencia@cecar.edu.co

2 Especialista en Derecho Procesal Civil. Coordinadora del Centro de Conciliación del Consultorio Jurídico de la Facultad de Derecho y Ciencias Políticas de la Corporación Universitaria del Caribe "CECAR". Abogada y Conciliadora en Derecho, Email: nubia.valdelamart@cecar.edu.co

3 Especialista en Derecho Procesal Civil y Gerencia de la Hacienda Pública. Estudiante del programa de Economía de la Universidad de Sucre. Abogado y Conciliador en Derecho. Funcionario judicial y Docente Universitario. Email: cristian.perezav@cecar. edu.co 

en derecho en el distrito judicial de Sincelejo, Colombia...

a la materialización del derecho de acceso a la justicia mediante la conciliación extrajudicial en derecho es idóneo, eficaz y de gran utilidad para la comunidad, toda vez que el análisis estadístico denota que el porcentaje de audiencias realizadas en las diferentes áreas del derecho, en las que el centro tiene competencia, es mayor a las demandas presentadas ante los juzgados, por los mismos usuarios del centro.

Palabras clave: Conflicto, Justicia, MASC, Conciliación, Centro de Conciliación.

\section{Abstract}

The extrajudicial conciliation in law, held at the Conciliation Center is a tool to ensure effective access to the administration of justice, in this investigation described and conceptualized the extrajudicial conciliation as a fundamental right, its history, existence and competence of the centers in the judicial district of Sincelejo. It was developed based on a methodological design organized in different phases, mainly an analysis of the statistics of the Conciliation Center of the Corporación Universitaria del Caribe CECAR, during the years 2016 and 2017, which allowed the realization of the results and conclusions, identifying that the contribution of CECAR's Conciliation Center to the materialization of the right of access to justice through extrajudicial conciliation in law is ideal, effective and very useful for the community, since the statistical analysis shows that the percentage of hearings held in the different areas of law, in which the center has competence, is greater than the claims filed before the courts, by the users of the center themselves.

Keywords: Conflict, Justice, Alternate Dispute Resolution Mechanism, conciliation, conciliation centre.

\section{Introducción}

Los Centros de Conciliación de los Consultorios Jurídicos de las Facultades de Derechos de las Universidades, en su objeto de creación tienen una función de proyección social, en los distintos distritos judiciales donde se encuentren ubicados; la proyección social consiste en acceder a la problemática de la cotidianidad en general, especialmente a la población de escasos recursos, y proporcionarles una pronta solución. Es por esta razón que el presente capítulo de libro pretende desarrollar ¿cuáles son 
los aportes del Centro de Conciliación de CECAR a la materialización del derecho de acceso a la justicia mediante la conciliación extrajudicial en derecho en el distrito judicial de Sincelejo (Colombia), durante los años $2016-2017$ ?

Para el desarrollo del presente capítulo se trazó como objetivo general identificar cuáles son los aportes del Centro de Conciliación de CECAR a la materialización del derecho de acceso a la justicia mediante la conciliación extrajudicial en derecho en el distrito judicial de Sincelejo (Colombia), durante los años 2016 - 2017. Para darle cumplimiento al objetivo anteriormente descrito se diseñaron tres objetivos específicos, los cuales son conceptualizar el Derecho de acceso a la justicia desde la ley y la jurisprudencia, describir la figura de la conciliación extrajudicial en derecho desde los centros de conciliación y el análisis de las estadísticas del Centro de Conciliación durante los años 2016 - 2017.

El capítulo de libro desarrollado es de gran relevancia, pertinencia e impacto debido a que sus resultados van dirigidos a socializar el aporte del Centro de Conciliación de la Facultad de Derecho y Ciencias Políticas de la Corporación Universitaria del Caribe CECAR, a toda la comunidad en general. A lo largo de esta investigación trataremos de culturizar a la población del distrito judicial de Sincelejo y zonas aledañas, sobre la gran importancia de solucionar sus conflictos de manera pacífica, garantizando así el derecho de acceso a la administración de justicia de manera ágil, voluntaria y gratuita, además contribuyendo en la descongestión de los despachos judiciales. El impacto de esta investigación se medirá a partir del análisis estadístico de número de casos y solicitudes atendidas por el Centro de Conciliación durante el periodo establecido en la presente investigación.

En cuanto a la utilidad que representará la investigación para el Centro de Conciliación de la Corporación Universitaria del Caribe-CECAR, es identificar los aportes del Centro a la materialización del derecho de acceso a la justicia mediante la conciliación extrajudicial en derecho en el distrito judicial de Sincelejo (Colombia), durante los años 2016 2017. También permitirá hacer un seguimiento del trabajo realizado, con la finalidad de medir la calidad y efectividad del servicio prestado, así mismo, será útil puesto que dentro de sus resultados se dejará un instrumento consistente en una matriz donde se organizará toda la información y esta permitirá analizar más fácilmente la efectividad y el aporte del centro en los tramites conciliatorios que en él se desarrollan y podrá ser utilizada para el análisis de futuras investigaciones. 
Por otra parte, en lo atinente a la utilidad para los investigadores, el desarrollo del presente capitulo implicará un crecimiento en el ámbito personal, profesional y académico, permitiéndonos razonar sobre la figura de la conciliación extrajudicial en derecho, y además nos servirá para nuevas competencias y experiencias en el campo de la investigación.

\section{Metodología}

El diseño metodológico del presente capitulo es de naturaleza mixta, en el entendido que se realizará un estudio cualitativo y cuantitativo, con un nivel hermenéutico. La investigación es de tipo cualitativa porque se pretende describir cual es el aporte del Centro de Conciliación de CECAR a la materialización del derecho de acceso a la justicia mediante la conciliación extrajudicial en derecho en el distrito judicial de Sincelejo (Colombia), durante los años 2016-2017, así mismo, es de tipo cuantitativo porque para darle cumplimiento al objetivo general en ejecución de los objetivos específicos, se realizará un análisis estadístico a través del cual determinaremos cuantas solicitudes, acuerdos, constancias de inasistencias, no acuerdos, retiros de solicitudes y otros resultados se obtuvieron durante el periodo en mención en el Centro de Conciliación de CECAR.

Por otro parte, esta investigación es de carácter jurídico con un enfoque epistemológico. Es jurídica porque las fuentes principales objeto de análisis son normatividades que rigen el derecho de acceso a la administración de justicia, la conciliación extrajudicial en derecho, la competencia de los Centros de Conciliación de Consultorios Jurídicos (Ley 270 de 1996, Ley 446 de 1998 , ley 640 de 2001 y demás normatividad relacionada), además, tiene un enfoque epistemológico porque se realizará un análisis estadístico que se interpretara y describirá a lo largo del desarrollo de la presente investigación, a partir de datos existentes en el archivo del Centro de Conciliación del Consultorio Jurídico de CECAR.

Para darle cumplimiento a los objetivos específicos trazados en la presente investigación se ha planteado el siguiente diseño metodológico:

- Fase 1: Para darle cumplimiento al objetivo específico número uno se realizará un rastreo bibliográfico para conceptualizar el acceso de administración de justicia como un derecho fundamental, adicionalmente se mencionarán distintas alternativas que permita establecer la evolución en relación al tema establecido.

- Fase 2: En cuanto al objetivo específico número dos se realizará un rastreo bibliográfico para conceptualizar la figura de la 
conciliación extrajudicial en derecho, posteriormente se diseñará una matriz de información sobre la existencia, competencia de los centros de conciliación y funcionarios habilitados para conciliar.

- Fase 3: Finalmente para lograr el objetivo específico número tres, se realizará la revisión y análisis de las estadísticas del Centro de Conciliación del Consultorio Jurídico de CECAR durante los años 2016 y 2017, en cuanto a los resultados obtenidos (acuerdo total, acuerdo parcial, no acuerdo, inasistencias, fijación de nueva fecha y retiro de la solicitud), la revisión de los seguimientos a los acuerdos conciliatorios suscritos en el centro para verificar el cumplimiento de las actas. Los resultados se reflejarán a través de unas graficas de información que nos permitirán determinar el aporte del Centro de Conciliación de CECAR a la materialización del derecho de acceso a la justicia, en el distrito judicial de Sincelejo (Colombia), durante los años 2016 - 2017.

\section{Fuente de análisis}

Dentro de las fuentes que utilizadas se encuentran las estadísticas de los años 2016 y 2017, los seguimientos a los acuerdos conciliatorios celebrados en los periodos objetos de la investigación, los nuevos seguimientos que se realizaran para verificar el cumplimiento de dichos acuerdos, el libro de expedición de primera copia para demanda, documentos estos propias del Centro de Conciliación del Consultorio Jurídico de la Facultad de Derecho y Ciencias Políticas de CECAR. También se tendrán en cuenta otras fuentes como libros, capítulos de libro, trabajos de grado y demás producciones bibliográficas referentes al tema estudiado, en general se utilizará todas las leyes, los decretos y jurisprudencias existentes de la materia objeto de investigación.

\section{Antecedentes}

Es de gran importancia destacar la evolución histórica de la conciliación iniciando desde el derecho colombiano para posteriormente puntualizar la evolución histórica Internacional. La conciliación es tan antigua como lo es la humanidad y el derecho ubi societas ibi jus (donde hay sociedad, hay derecho) (Garcia, 2013), la figura de la conciliación en Colombia tiene su origen después de la independencia, se da inicio a ella con la expedición de la Ley del 13 de mayo de 1825, propuesta por Simón Bolívar, en la que se reglamentó el requisito de procedibilidad para demandar en la jurisdicción 

en derecho en el distrito judicial de Sincelejo, Colombia...

civil, eclesiástica o militar, un suceso que marco la figura de la conciliación fue: que el alcalde se enterará de las razones que aleguen y procurará transigirlas y avenirlas entre sí por las medidas suaves de una conciliación amigable (López, 2002).

Luego de realizar un rastreo bibliográfico se precisó en acontecimientos de gran relevancia para el derecho internacional para la figura de la conciliación, algunos de esos momentos históricos destacados por la jurisprudencia, (Corte Suprema de Justicia. Sentencia de Casación del 15 de diciebre de 1948), fueron:

- En Roma: Una parte de la doctrina encuentra antecedentes en la ley de las doce tablas, manifestando que uno de sus textos daba fuerza obligatoria a lo que convinieran las partes intervinientes al momento de ir a juicio.

- Legislación Portuguesa: Con el código Manuelino, a partir del año 1521, se ordenó acudir a la conciliación antes de cualquier otra instancia.

- En España: Con la ley de enjuiciamiento civil de 1855, se le dio carácter obligatorio al trámite de conciliación para poder iniciar un juicio.

- Legislaciones como la francesa, la española, la italiana, la alemana y la argentina establecen la conciliación como obligatoria antes de acudir a otras instancias judiciales.

En Colombia a partir de la Constitución de 1991 (Constitución Politica de Colombia), consagrado como un Estado social de derecho, se incluyó la conciliación como mecanismo alternativo de solución de conflictos, principalmente en su artículo 116 presentó el marco legal para la existencia de otras formas de justicia diferentes a la ordinaria o tradicional, como una salida práctica, efectiva y alterna para coadyuvar en la solución de conflictos familiares, civiles y comerciales. Nuestra carta fundamental hace manifiesta la conciliación, así: Los particulares pueden ser investidos transitoriamente de la función de administrar justicia en la condición de jurados en las causas criminales, conciliadores o en la de árbitros habilitados por las partes para proferir fallos en derecho o en equidad, en los términos que determine la ley. 
Tabla 1

Normativas en Colombia

\section{NORMATIVI- DAD}

\section{TEMA QUE REGULA}

Ley 23 de 1991

Por medio de la cual se crean mecanismos para descongestionar los Despachos Judiciales, y se dictan otras disposiciones.

Ley 446 de 1998 ... se modifican y expiden normas del Código Contencioso Administrativo y se dictan otras disposiciones sobre descongestión, eficiencia y acceso a la justicia.

Ley 640 de Por la cual se modifican normas relativas a la conciliación y 2001 se dictan otras disposiciones.

Ley 270 de Estatutaria de la Administración de Justicia (reformada por 1996 la Ley 1285 de 2009)

Ley 1395 de Por la cual se adoptan medidas en materia de descongestión 2010 judicial.

Ley 1563 de 2012

Por medio de la cual se expide el Estatuto de Arbitraje Nacional e Internacional y se dictan otras disposiciones.

Ley 1564 de 2012 Por medio de la cual se expide el Código General del Proceso y se dictan otras disposiciones.

Decreto 1818 Por medio del cual se expide el Estatuto de los mecanismos de 1998 alternativos de solución de conflictos.

Por el cual se reglamenta la conciliación extrajudicial

Decreto 2511 contencioso administrativa y en materia laboral previstas de 1998 en la Parte III, Título I, capítulos 1, 2 y 3, Secciones 1, 2 y 3 de la Ley 446 de 1998, y en los artículos 19, 21 y 22 del Código Procesal del Trabajo.

Decreto 1214

Por el cual se establecen funciones para los Comités de de 2000 Conciliación de que trata el artículo 75 de la Ley 446 de 1998 y se dictan otras disposiciones.

Decreto 2771 Por medio del cual se reglamenta el artículo 42 de la Ley de 2001 640 de 2001.

Decreto 1829 Por el cual se reglamentan algunas disposiciones de las de 2013 Leyes 23 de 1991, 446 de 1998, 640de 2001 y 1563 de 2012.

Decreto 1069 Por medio del cual se expide el Decreto Único Reglamentario de 2015 del sector Justicia y del Derecho

Elaborada por los investigadores en mención, con base en las leyes vigentes en materia de Conciliación en Colombia. 
La tabla anteriormente relacionada hace alusión a las leyes y decretos que dieron estructura a todos los Mecanismos Alternativos de Solución de Conflictos - MASC, que conforman la llamada justicia alternativa. Las leyes 446 de 1998, 640 de 2001, 1563 de 2012 y el Decreto 1069 de 2015, definen y regulan actualmente la conciliación, el arbitraje, la amigable composición, la transacción y la negociación.

Para adentrar en el tema objeto de investigación es necesario tener claridad frente a la definición de algunos conceptos que serán tratados a lo largo del capítulo, ellos son:

Conflicto: Debeentenderse por conflicto las diferencias o controversias entre una o más personas, ocasionadas en razón a diferencia de intereses contrapuestos, desigualdades e inconformidades; ocasionalmente los seres humanos pensamos o afirmamos que los conflictos son de naturaleza negativa, sin embargo, no todos los conflictos obedecen a un estado negativo, pueden ser constructivos.

Justicia: Es el conjunto de valores que nos permite juzgar respetando la verdad y dándole a cada uno lo que le corresponde.

Mecanismos Alternativos de Solución de Conflictos: Es considerado como un conjunto de herramientas a través del cual las personas pueden gestionar sus diferencias, de manera ágil, eficiente, pacífica, eficaz, voluntaria, y con plenos efectos legales; de ahora en adelante MASC.

Conciliación: La conciliación es un mecanismo de resolución de conflictos a través del cual, dos o más personas gestionan por sí mismo la solución de sus diferencias, con la ayuda de un tercero neutral y calificado, denominado conciliador (Ley 446, 1998).

Centro de Conciliación: Es aquel autorizado por el Ministerio de Justicia y del Derecho para que preste el soporte operativo y administrativo requerido para el buen desarrollo de las funciones de los conciliadores (Decreto 1829,2013$)$.

\section{Derecho de acceso a la justicia desde la ley y la jurisprudencia colombiana}

Desde el inicio de los tiempos y a través de la historia, los seres humanos han vivido en conflicto, entendiendo por conflicto una situación de guerra, lucha, oposición de intereses en que las partes no ceden, choque o colisión de derechos o pretensiones (Cabanellas, 1976); Igualmente, las personas siempre han intentado y tratado de hacer todo lo posible para solucionar las diferencias surgidas entre ellas. En nuestro país, con miras de 
resolver los conflictos entre las personas se ha creado un puente entre los ciudadanos con conflictos o controversias y la solución de los mismos, este es el derecho de acceso a la administración de justicia.

Antes de hablar del derecho de acceso a la justicia, es preciso recordar el concepto de justicia, esta es definida por la Real Academia Española (2018) como: el principio moral que implica dar a cada quien lo que le corresponde o pertenece, o podríamos ampliar el mismo concepto conforme a la definición del Congreso de Colombia, el cual mediante la Ley Estatutaria de la Administración de Justicia (Ley 270., 2009), define la justicia como un valor superior establecido en la Constitución Política que debe orientar la acción del Estado y debe asegurar la efectividad de los derechos fundamentales, dentro del marco del Estado Social de Derecho, y alcanzar la convivencia pacífica entre los ciudadanos, y que genera responsabilidad de quienes están encargados de ejercerla.

A partir de la Constitución de 1991, donde se da el paso al Estado Social de Derecho, desde su preámbulo se consagró a la justicia como un valor supremo, de esa manera, el acceso a la administración de justicia adquiere la connotación de un derecho fundamental, enmarcado especialmente en el artículo 228 que la define como una función pública, determinando que las decisiones que en virtud de ella se tomen son independientes, y que todas las actuaciones que se den en ocasión a ella serán públicas y permanentes con las excepciones que la ley determine, siempre prevaleciendo el derecho sustantivo sobre el adjetivo (Constitución Política de Colombia , 1991).

Por su parte gracias al artículo 229 de la Carta política se garantiza el derecho que tiene toda persona para acceder a la administración de justicia y se deja abierta la posibilidad de que la ley determine en qué casos las personas podrán acceder a este derecho sin la representación de abogado, lo cual ya ha sido regulado por la ley, existiendo hoy la posibilidad de que las personas actúen en nombre propio, en los asuntos que así se ha determinado (Constitución Política de Colombia , 1991).

Igualmente, desde la jurisprudencia, se ha ampliado el concepto constitucional del derecho de acceso a la administración de justicia manifestando que este es la facultad que se le ha concedido a todas las personas residentes en Colombia para que, en condiciones de igualdad ante los órganos jurisdiccionales, protejan o busquen el restablecimiento de sus derechos e intereses legítimos, respetando los procedimientos previamente establecidos y con total cumplimiento de las garantías sustanciales y procedimentales previstas en las leyes, buscando 

en derecho en el distrito judicial de Sincelejo, Colombia...

mantener la integridad del orden jurídico (Corte Constitucional de Colombia. Sentencia C - 1083, 2005).

Conforme a lo anteriormente mencionado, se puede decir que el Derecho de Acceso a la Administración de Justicia constituye una herramienta para proteger el conjunto de los derechos humanos reconocidos constitucionalmente, siendo la garantía que tenemos las personas para que se aplique justicia, independiente de su naturaleza, condiciones y perjuicios. Es el reconocimiento o garantía que tiene todo ser humano de no tomar la justicia por su propia mano, sino de poder contar con el amparo del Estado para, a través del acceso a la administración de justicia, poder solucionar sus conflictos de manera legal, pacífica con igualdad de cada una de las partes con diferencia en sus intereses.

En este sentido, el estado colombiano es quien está obligado a proteger y garantizar una igualdad material, colocando en equidad y en el mismo punto de equilibrio a todo el que pretende reclamar sus derechos, siendo su deber garantizar el derecho a la administración de justicia, por ello debe permitir el fácil acceso a la jurisdicción a todos los ciudadanos, cuando consideren que sus derechos están siendo negados, violados o amenazados, para ello debe proporcionar la solución bajo los preceptos del ordenamiento vigente; solución que debe tener como resultado el fin al conflicto de manera justa, siempre fundamentado en el derecho sustancial, con una valoración e interpretación efectiva de la constitución y la ley.

Es preciso decir, que el acceso a la justicia es un derecho fundamental de aplicación inmediata, tal y como ha sido reconocido por la Corte Constitucional, el derecho a acceder a la justicia ayuda de manera efectiva al cumplimiento material de los fines esenciales e inmediatos del Estado, tales como son garantizar un orden político, económico y social justo, instar la convivencia pacífica, procurar por el respeto a la legalidad y a la dignidad humana y asegurar la protección de las personas en su vida, honra, bienes, creencias y demás derechos y libertades dadas desde la constitución y el bloque de constitucionalidad.

El fundamento del derecho a la tutela judicial efectiva también se encuentra consagrado en las normas de derecho internacional, especialmente en la Declaración Universal de Derechos Humanos, la Convención americana de Derechos Humanos y el Pacto Internacional de Derecho Civiles y Políticos, por mencionar algunos. Conforme a la Declaración Universal de Derechos Humanos en su artículo 10, todas 
las personas poseen el derecho de ser escuchadas, en forma pública y con justicia, por un tribunal u órgano judicial que sea imparcial e independiente, para la protección de sus derechos y el establecimiento de obligaciones o para examinar cualquier acusación contra ellas en materia penal, todo lo anterior en total igualdad de condiciones. Igualmente, en el mismo documento internacional, en el artículo 11.1 se determina que toda persona que sea acusada de delito goza de la presunción de su inocencia mientras no se demuestre lo contrario, en un juicio público en el que se aseguren todas las garantías para su defensa (Corte Constitucional de Colombia. Sentencia C-426, 2002).

Por su parte, la Convención Americana (1969), en su Artículo 25, enuncia: que todas las personas tienen derecho a un recurso simple, ágil y efectivo ante los jueces o tribunales, que la proteja de todos aquellos actos que transgredan sus derechos fundamentales constitucional, legal o convencionalmente reconocidos, aun cuando tal violación sea cometida por personas que actúen en ejercicio de sus funciones oficiales.

\section{Alcance y efectos del derecho a la administración de justicia}

No debemos limitar el derecho de acceder a la administración de justicia, a la potestad de acudir físicamente ante los órganos de la Rama Judicial o las entidades que poseen ciertas funciones jurisdiccionales en virtud a la competencia conferida en el artículo 116 de la Constitución Política de Colombia, sino ampliar esta posibilidad de poner en marcha el aparato judicial y de que la autoridad competente resuelva de fondo el asunto que le ha sido presentado, respetando el debido proceso y llevándose a cabo de manera oportuna (Corte Constitucional de Colombia. Sentencia C-985 de 2005.)

En esa medida, el derecho a la administración de justicia no se entiende concluido con la simple solicitud o el planteamiento de las pretensiones procesales ante las respectivas instancias judiciales; sino que debe ser efectivo (Corte Constitucional de Colombia. Sentencia C-037 de 1996), por lo cual el mismo no cumple su propósito con la sola consagración formal de recursos y procedimientos, sino que necesita que éstos sean realmente idóneos y efectivos, implicando esto que no es suficiente que esté establecido constitucional o legalmente o con que sea formalmente posible, sino que se requiere que sea materialmente apto para determinar si se ha incurrido o no en una afectación a los derechos humanos y en caso de que si se haya transgredido, brindar lo necesario para remediar la situación o para reparar el daño. 

en derecho en el distrito judicial de Sincelejo, Colombia...

En este mismo sentido la Corte Interamericana de Derechos Humanos se ha pronunciado al interpretar el artículo 25.1 de la Convención Americana de Derechos Civiles y Políticos para definir cuándo no existe recurso judicial efectivo, expresando que no pueden tenerse como efectivos aquellos recursos que sean ilusorios, lo cual sucede, verbi gracia, cuando la rama Judicial no posee la independencia suficiente para decidir de forma imparcial, cuando faltan los mecanismos para cumplir sus decisiones o cuando se da un retardo injustificado en la decisión; o, por cualquier causa, no permita al presunto afectado acceder al recurso judicial (Corte IDH. Opinión Consultiva OC-9/87 del 6 de octubre de 1987, Serie A No. 9, párr. 24).).

En una sociedad democrática como lo es la colombiana, sabemos que debe garantizarse el derecho de acceso a la justicia, pero este derecho se pude ver afectado cuando por motivos económicos o sociales, las personas son excluidas o están en desventaja ante los sistemas de justicia, como muchas veces ocurre en nuestro país en la práctica, cuando no se garantiza la igualdad de condiciones para que los habitantes de todo el territorio colombiano puedan acceder a las autoridades para la protección de sus derechos.

\section{Distintas alternativas de acceso a la administración de justicia}

A pesar de que el derecho a acceder a la justicia está protegido en Colombia desde la Constitución, encontramos que a gran parte de la población del país no se le garantiza efectivamente este derecho, principalmente por las siguientes causas: la desconfianza en los sistemas de justicia, el gran porcentaje de personas en condición de vulnerabilidad, los escases servicios jurídico gratuitos e idóneos, el desconocimiento de los derechos y los trámites para garantizarlos, entre otros.

Por lo anterior, se hace necesario garantizar un amplio acceso a la administración de justicia, lo cual podría ser posible a través del trato igualitario de quienes acuden en busca de la misma, la representación legal adecuada y gratuita para aquellas personas que no tienen los recursos económicos, la debida información y orientación de los tramites y procedimientos para la protección de los derechos e intereses de las personas, la sensibilización de quienes administran justicia, la implementación de estrategias para promover la protección de los derechos humanos y crear programas de gestores jurídicos. De esta forma se podría ver mejor materializado, efectivamente, el derecho de acceso a la administración de justicia. 
Todos los sujetos tenemos la posibilidad de ser parte en un proceso, pero no debemos mirar la administración de justicia solo desde ese punto de vista, es decir no quedarnos en que solo podemos acceder a la justicia a través de un proceso judicial, acudir ante un juez para que este sea quien nos ampare nuestros derechos, porque también pueden ser amparados haciendo uso de ciertas herramientas como los Mecanismos Alternativos de Solución de Conflictos - MASC, como lo son la conciliación, el arbitraje, la amigable composición, la transacción y la negociación.

La aplicación de mecanismos como la conciliación extrajudicial en derecho tiene muchísimas ventajas en comparación con los medios tradicionales de administración de justicia, pues no solo se debe mirar como instrumento para solucionar el número de casos para descongestionar el aparato judicial, sino que se busca con el desarrollo de la audiencia que las partes lleguen a un acuerdo que sea satisfactorio y les permita a ambas partes ganar por igual, logrando una eficiencia de la administración de justicia, ahorrando mucho tiempo y dinero, resolviendo sus controversias de manera ágil y efectiva con la ayuda de un tercero neutral calificado, y sobre todo con la implantación de este mecanismo generamos una cultura de paz.

\section{La conciliación extrajudicial en derecho como mecanismo alternativo de solución de conflictos}

En periodos de tiempo moderno, las sociedades de distintas maneras han alternado en particulares formas de administrarse justicia a sí mismas. En diversidad de maneras estas han constituido mecánicas clásicas de proveerse justicia social, estableciendo esquemas procesales ampliamente basados en el ritualismo y la inflexibilidad legal, gestándose así en su mayoría los sistemas clásicamente dispositivos de administración de justicia, que recogen en la actualidad gran parte de las estructuras judiciales de los estados. Los anteriormente mencionados sistemas de administración de justicia, apelan a principios rígidos de formalidad procesal que permean todo el trámite, pero que nacen de la racionalidad misma de la humanidad por entregar justicia con garantías a quienes la peticionan.

Es entonces la racionalidad humana la que da forma a los sistemas de administración de justicia, dejando a un lado aspectos de la primitiva violencia en camino a la obtención de derechos. Siendo criterio ordenador de la administración de justicia, debemos mencionar que este es a la vez una aptitud presente en las partes que asumen un conflicto y 

en derecho en el distrito judicial de Sincelejo, Colombia...

persiguen su resolución. De manera alternativa a los sistemas clásicos de administración de justicia, encontramos formas simplificadas de resolver los distintos conflictos en la sociedad como la conciliación extrajudicial en derecho. A la cual debemos decir que, en conjunto con los demás mecanismos alternativos de resolución de conflictos, plantean un camino alternativo para la consecución de subterfugio a los problemas que surgen en medio de la convivencia en sociedad. (De Zan, 2004)

Realizando un recorrido histórico simple desde los inicios de la humanidad y observando su devenir en relación a la vida en comunidades; se debe partir por pasados primitivos de comunidades nómadas bajo convivencia escasa, que, por imaginario colectivo actual, se perciben como basadas en la violencia y la imposición de la fuerza como dispositivo definidor de las disputas que pudieren surgir estando en convivencia, viniendo a ser la realidad algo más compleja que ello. Y aterrizando en etapas posteriores de la humanidad que sientan bases a lo que conocemos como modernidad, en el sentido de la tradición romana de occidente, del establecimiento de la norma positiva vigente, como estatuto regulador de las sociedades modernas y su convivencia social; forjando así los ya conocidos sistemas de administración de justicia.

Nuestra corte concibe la administración de justicia partiendo de esta como una función misional del estado y derecho de todo ciudadano colombiano decantando por vía de jurisprudencia su concepto, por lo que se ha establecido que esta es una función pública que debe ser entendida como un complemento del derecho constitucional del libre acceso a la jurisdicción, gracias al cual, las personas tienen la facultad de ser parte en un proceso promoviendo la actividad jurisdiccional que finalice con una decisión judicial referente a las pretensiones propuestas. Ciertamente, el artículo 229 Superior, (Constitución Politica de Colombia, 1991), reconoce a todas las personas el derecho a obtener tutela judicial efectiva por parte de los jueces y tribunales que integran la administración de justicia, garantía que entraña la posibilidad de acudir libremente a la jurisdicción siendo parte en un proceso promoviendo la actividad jurisdiccional que concluya con una decisión final motivada, razonable y fundada en el sistema de fuentes (art. 230 de la C.P). También implica obviamente la existencia de pretensiones legítimas en cabeza de quienes accionan el aparato de la justicia. (Corte Constitucional de Colombia. Sentencia C-893 de 2001).

Lo anterior, puede constar como antecedente histórico de carácter general a lo que hoy llamamos la conciliación extrajudicial en derecho 
como mecanismo alternativo de resolución de conflicto, que para los fines de esta investigación, debemos destacar que Colombia persigue la tradición romana, sentando en norma positiva esta racional forma de dar fin por parte los ciudadanos a sus conflictos; como lo explica Pablo Tomas Silva Mariño (2009) al aducir que "contrario a la creencia normal, la conciliación no es una figura moderna en el Derecho colombiano", resaltando sí, un intenso desarrollo normativo y practico social, a partir de la consagración de la Constitución política Colombiana de 1991, pero que esta está presente en Colombia desde los inicios de su vida republicana en el siglo XIX con la promulgación de la ley 13 de 1825.

Podemos agregar, que, a partir de la promulgación de la Constitución Política de 1991, en Colombia, en referencia a la conciliación extrajudicial en derecho se conceptualiza un marco normativo disperso, que se aglutina finalmente con la ley 640 de 2001 y establece un concepto claro de esta, que recoge su importancia a nivel institucional, procesal y social. Es entonces, la conciliación parte de un sistema alternativo de administración de justicia en medio del juego de la ciudadanía y a su vez, un mecanismo basado en criterios de racionalidad, que permite a partir de la intervención de un tercero, neutral, imparcial y calificado, denominado conciliador, la solución del conflicto que se pudiere presentar entre las personas, quienes auto gestionan la solución de sus disputas conforme con la guía e intervención de este mencionado tercero.

Se le llama extrajudicial, porque partiendo de ser una forma alternativa a los sistemas de justicia tradicional, se surte por fuera de los procedimientos judiciales del sistema ordinario y general de administración de justicia en Colombia; aunque cabe decir, que está igualmente presente dentro de estos, como etapa procesal correspondiente y necesaria de la mayoría de formas que conoce la justicia ordinaria. La conciliación extrajudicial en derecho, es entonces punto intermedio entre la ciudadanía y la función de administrar justicia por parte del estado, puesto que permite en primer lugar delegar la tan importante labor de integrar equidad ciudadana por medio de la justicia y descongestiona los ya atestados estrados judiciales.

En Colombia, se debe decir, que hasta entrado el siglo XXI, aún se tenía por costumbre judicializar los problemas que surjan a diario; esto, a causa de la confianza legítima pero recelada en los medios que representa el estado y el poco merito a lo que no implique coercitividad y presencia de elevados funcionarios que por forzada labor impartan solución a los problemas ciudadanos. Viene ahora a ser la conciliación 

en derecho en el distrito judicial de Sincelejo, Colombia...

extrajudicial en derecho, modificador de esa concepción tradicional de judicialización de los problemas por más comunes y simples que se tengan, agitando las bases mismas de la costumbre ciudadana de cómo solucionar sus problemas y cambiando la percepción general de esto al día de hoy. El establecimiento de la conciliación extrajudicial en derecho, como requisito de procedibilidad, consideramos, constituye una apuesta importante por modificar la forma en que racionalmente le impartimos solución a los problemas; lo es así porque el estado conmina por intermedio de la ley a que se recurra al dialogo y la gestión simple del conflicto en espacios preparados para ello, en compañía de personal igualmente calificado y con la confianza legítima de que allí también se consigue justicia.

Los centros de conciliación y los funcionarios facultados para el ejercicio como conciliadores, se constituyen en medios de facilitación para la solución de problemas ciudadanos, cuyas bases competitivas se encuentran establecidas en la misma ley. Es así que el legislador instituye límites no cerrados, sino más bien amplios de las competencias de los centros de conciliación, cercándose solamente en temas de derecho administrativo y laboral, que, bajo pronunciamiento expreso de la corte constitucional, restringió sus facultades y por consecuencia la competencia de los centros de conciliación y de algunos funcionarios del estado. En el ámbito territorial, donde se imparte análisis y se esquematizan los datos que resultantes de esta investigación, el departamento de Sucre y el municipio de Sincelejo en particular, existe una amplia oferta de centros de conciliación y funcionarios públicos facultados por la misma ley para el conocimiento de la conciliación extrajudicial en derecho.

Centros de conciliación de naturaleza privada en la región, se encuentran en primer lugar, aquellos dependientes de los consultorios jurídicos de facultades de derecho de las universidades que contengan dentro de su oferta académica el programa de derecho, como cumplimiento de un requisito que ley misma establece a efectos de la formación de los estudiantes de derecho y de la proyección social que es inherentes a estas instituciones de carácter privado; es así como encontramos de manera general en Colombia un total de ciento treinta y cuatro (134) Consultorios jurídicos y a partir de allí, los Centros de Conciliación anexos a estos (Dirección de Metodos alternativos de solución de conflictos, 2018); de los cuales en la zona del distrito judicial de Sincelejo, solo existen dos (2), siendo los Consultorio Jurídico 
de la Corporación Universitaria del Caribe - CECAR y la Corporación Universitaria Antonio José de Sucre - CORPOSUCRE, los correspondientes.

Del lado de la iniciativa privada en el distrito judicial de Sincelejo, encontramos el Centro de Conciliación de la Fundación Liborio Mejía y el Centro de Conciliación y arbitraje "Centro Profesional de Justicia". Se debe destacar, que la Cámara de Comercio de la ciudad posee su propio centro de Conciliación, que completa la oferta local de entidades que conocen de la conciliación extrajudicial en conjunto con el resto de entidades públicas facultadas expresamente por la ley para el conocimiento por materias de este mencionado mecanismo alternativo; tales como, los notarios del circulo notarial de Sincelejo, la Defensoría del Pueblo, la Personería Municipal, las comisarías de familia, las oficinas regionales del Instituto Colombiano de Bienestar Familiar - ICBF, el Ministerio del trabajo, de competencia exclusiva para presidir conciliaciones en materia de derecho laboral y las procuradurías judiciales correspondientes de la región, de competencia exclusiva para el conocimiento de conciliación extrajudicial en materia de derecho administrativo.

Ahora bien, de las iniciativas particulares de centros de conciliación en el distrito judicial de Sincelejo, encontramos que estas a su vez se diferencian en relación a las competencias que conforme la ley establece; partiendo de la cuantía que con anterioridad la ley misma determina general y residualmente por intermedio del Código General del proceso en su artículo 25; sirviendo como criterio diferenciador del servicio que prestan los centros de conciliación en la ciudad. Es así que los centros de Conciliación adscritos a los Consultorios jurídicos de las facultades de derecho tanto de la Corporación Universitaria del Caribe - CECAR, como de la Corporación Universitaria Antonio José de Sucre - CORPOSUCRE, conocen de conciliaciones extrajudiciales en derecho exclusivamente sobre asuntos de mínima cuantía y más diferenciador aún resulta el hecho de que solo se atiende población de escasos recursos.

Resta entonces a las otras entidades presentes en el distrito judicial de Sincelejo, completar la oferta de centros de conciliación, que conozcan de conciliación extrajudicial en derecho de manera indistinta de su cuantía, en las que encontraremos al Centro de Conciliación Fundación Liborio Mejía, al Centro Profesional de Justicia y el centro de conciliación de la cámara de Comercio de esta ciudad. En la siguiente tabla ilustrativa; encontraremos un esquema simplificado por competencias, de los distintos centros y entidades que conocen de conciliación extrajudicial en derecho en el distrito judicial de Sincelejo, buscando instruir más acerca de las distintas 

en derecho en el distrito judicial de Sincelejo, Colombia...

rutas, por intermedio de las cuales los ciudadanos de esta comunidad pueden tener acceso a una completa administración de justicia, aclarando la labor que estas instituciones prestan a la comunidad.

Tabla 2

Centros y entidades que conocen de conciliación extrajudicial en derecho en el distrito judicial de Sincelejo

\begin{tabular}{|c|c|c|c|}
\hline $\begin{array}{l}\text { Naturaleza } \\
\text { Jurídica de } \\
\text { la entidad }\end{array}$ & $\begin{array}{l}\text { Competencia } \\
\text { por cuantías }\end{array}$ & Nombre de la entidad & $\begin{array}{l}\text { Asuntos de } \\
\text { que conoce }\end{array}$ \\
\hline Publica & $\begin{array}{l}\text { Mínima, } \\
\text { Menor y } \\
\text { Mayor }\end{array}$ & $\begin{array}{c}\text { Funcionarios habilitados: } \\
\text { Procuraduría, Defensoría } \\
\text { del Pueblo, Ministerio } \\
\text { de la protección social, } \\
\text { ICBF, Comisaria de familia, } \\
\text { personería, fiscalía. }\end{array}$ & $\begin{array}{l}\text { Familia, civil, } \\
\text { penal, laboral }\end{array}$ \\
\hline Privada & $\begin{array}{l}\text { Mínima, } \\
\text { Menor y } \\
\text { Mayor }\end{array}$ & $\begin{array}{l}\text { Funcionarios habilitados: } \\
\text { Notarias }\end{array}$ & Familia, civil \\
\hline \multirow[b]{2}{*}{ Privada } & Mínima & $\begin{array}{l}\text { Centros de Conciliación de } \\
\text { los Consultorios Jurídicos de: } \\
\text { La Corporación Universitaria } \\
\text { del Caribe - CECAR y La } \\
\text { Corporación Universitaria } \\
\text { Antonio José de Sucre - } \\
\text { CORPOSUCRE. }\end{array}$ & $\begin{array}{c}\text { Civil, familia, } \\
\text { policivo y } \\
\text { penal }\end{array}$ \\
\hline & $\begin{array}{l}\text { Mínima, } \\
\text { menor y } \\
\text { mayor. }\end{array}$ & $\begin{array}{l}\text { Centro de Conciliación } \\
\text { "Corporación centro } \\
\text { profesional de justicia: } \\
\text { conciliación, arbitraje y } \\
\text { amigable componedor", } \\
\text { Centro de Conciliación de } \\
\text { la Fundación Liborio Mejía, } \\
\text { y Centro de Conciliación y } \\
\text { arbitraje de la Cámara de } \\
\text { Comercio. }\end{array}$ & Civil, y familia \\
\hline
\end{tabular}

Nota: Elaborada por los investigadores en mención. Con base en los distintos centros y entidades que conocen de conciliación extrajudicial en derecho en el distrito judicial de Sincelejo. 
Análisis de las estadísticas del Centro de Conciliación durante los años $2016-2017$

Para identificar cuáles han sido los aportes del Centro de Conciliación del Consultorio Jurídico de la Corporación Universitaria del Caribe CECAR, a la materialización del derecho de acceso a la justicia mediante la conciliación extrajudicial en derecho en el distrito judicial de Sincelejo - Colombia, en los años 2016 y 2017, hemos analizado las estadísticas del Centro para estos dos periodos, las cuales han arrojado los resultados a continuación descritos.

Resultados generales años 2016 y 2017

Resultados generales 2016

\section{Resultados Generales 2016}

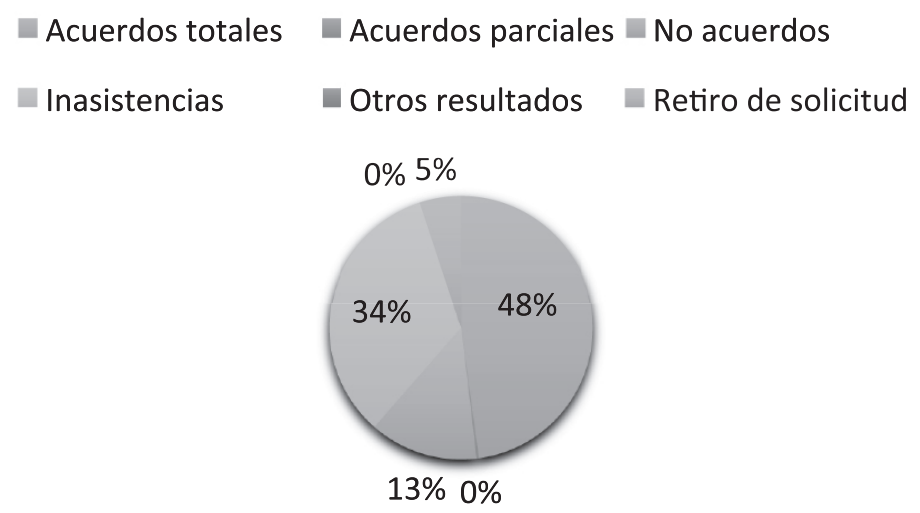

Gráfica 1. Resultados generales año 2016

Fuente: Elaborada por los investigadores en mención, de la información obtenida de la Coordinación del Centro de Conciliación del Consultorio Jurídico de CECAR, año 2016.

La gráfica 1, arrojó los resultados generales obtenidos durante el año 2016, se organizó de acuerdo a las estadísticas finales realizadas por la coordinación del Centro de Conciliación de CECAR, obteniendo como resultados 180 acuerdos totales, 126 inasistencias, 50 no acuerdos, 19 retiros de solicitudes y 1 acuerdo parcial, teniendo 376 resultados en general. A partir del análisis estadístico de la gráfica $\mathrm{N}^{\circ} 1$ el mayor porcentaje arrojado es de 180 acuerdos totales, correspondientes al $48 \%$ de los casos tramitados en el centro, lo que significa que en el año 

en derecho en el distrito judicial de Sincelejo, Colombia...

2016 el aporte del Centro de Conciliación fue bastante significativo para la población de Sincelejo y sus alrededores, logrando así la solución de diferentes conflictos de manera pacífica, gratuita y ágil, siendo los usuarios los protagonistas de sus propias soluciones sin necesidad de acudir a la jurisdicción ordinaria lo que denota el aporte significativo a la descongestión judicial y a una cultura de paz para nuestra sociedad.

\section{Resultados generales 2017.}

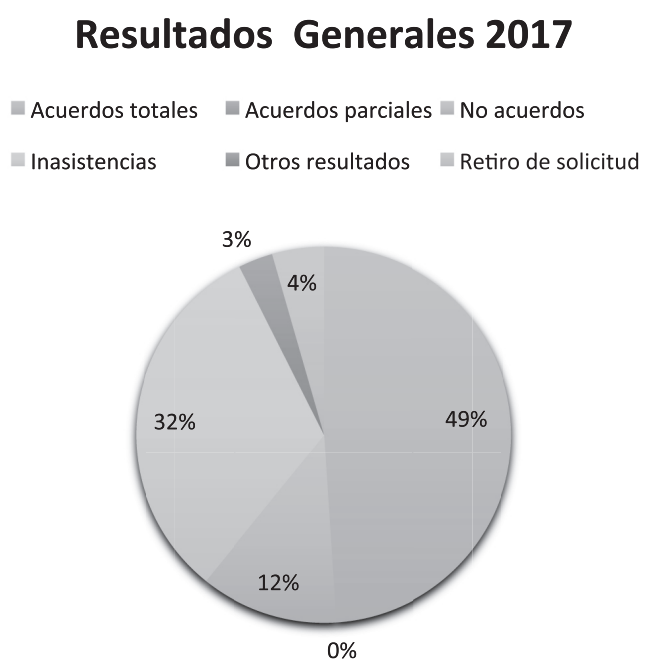

Gráfica 2. Resultados generales año 2017.

Fuente: Elaborada por los investigadores en mención, de la información obtenida de la Coordinación del Centro de Conciliación del Consultorio Jurídico de CECAR, año 2017.

En la gráfica 2, se pueden evidenciar los resultados generales obtenidos durante el año 2017, teniendo así un total de 337 casos atendidos, distribuidos en 165 acuerdos totales, 107 inasistencias, 40 no acuerdos, 15 retiros de solicitudes y 10 resultados diferentes. De la gráfica $N^{\circ} 2$, en comparación con la gráfica $N^{\circ} 1$, se puede concluir que aunque en el año 2016 se lograron 15 acuerdos más, en el año 2017 se mantuvo la tendencia de que los acuerdos tuvieran mayor porcentaje en relación con los demás resultados, con un 49\%, lo que significa que la actividad del Centro de Conciliación es de gran importancia para la ciudad de Sincelejo ya que muy posiblemente se evitó la presentación de 165 demandas durante ese año, disminuyendo así la congestión de los despachos judiciales y a su vez contribuyendo en el goce efectivo de 
acceder a la justicia de forma oportuna, ágil, gratuita y además las partes quedan con la satisfacción de resolver sus controversias de manera amigable y resultando ambos vencedores.

Los resultados correspondientes a inasistencias, retiros de solicitudes o lo que denominamos otros resultados, que en gran medida corresponden a desistimientos tácitos, obedecen generalmente a que una vez la parte convocada recibe la correspondiente citación a la audiencia de conciliación voluntariamente accede a solucionar el conflicto de manera directa y personal con la parte convocante, sin que necesidad de que este último continúe con el tramite conciliatorio, toda vez que resuelven directamente sus controversias.

Cabe resaltar que el Centro de Conciliación de CECAR, además de contar con el personal idóneo (abogados y estudiantes conciliadores) cuenta con un equipo interdisciplinario de psicólogos y trabajadores sociales que apoyan la labor del conciliador en el desarrollo de las audiencias de Conciliación, gracias al acuerdo de cooperación solidaria, que existe con el Centro de Familia de CECAR.

Adicionalmente con la atención prestada en los años 2016 y 2017 , más de 690 personas y sus familias, lograron solucionar sus conflictos, personas que no disponen de medios económicos para acceder al servicio de un abogado e instaurar un proceso judicial, aporte que denota la proactividad y contribución significativa del Centro de Conciliación de CECAR.

Solicitudes por materia, años 2016 y 2017.

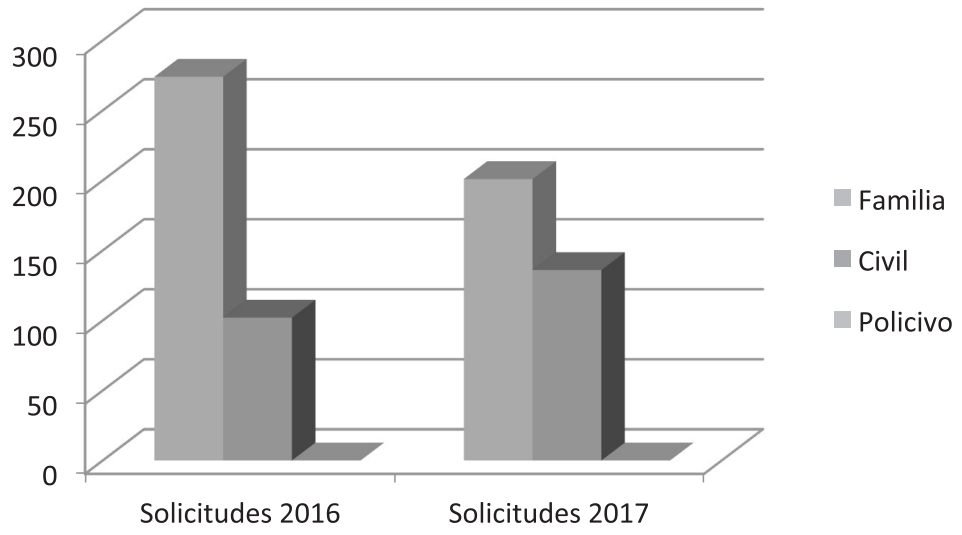

Gráfica 3. Solicitudes por materia, años 2016 y 2017.

Fuente: Elaborada por los investigadores en mención, de la información obtenida de la Coordinación del Centro de Conciliación del Consultorio Jurídico de CECAR, años 2016 y 2017. 

en derecho en el distrito judicial de Sincelejo, Colombia...

En la gráfica 3, se establecieron los asuntos en que más se agendaron audiencias de conciliación en dicho Centro, en cuanto al año 2016, en materia de familia, fueron: Fijación de cuota de alimentos, declaración de unión marital de hecho, aumento, disminución y ofrecimiento voluntario de cuota de alimentos, disolución de unión marital de hecho, custodia y cuidado personal, exoneración de cuota de alimentos, y regulación de visitas, respectivamente.

En materia Civil, son: Responsabilidad civil extracontractual, contrato de compraventa, mutuo, arrendamiento, responsabilidad civil contractual, reivindicatorio de dominio, cobro de sumas de dinero, responsabilidad médica.

Por su parte en el año 2017, los asuntos que se recepcionarón en materia de familia, de mayor a menor frecuencia fueron: Fijación de cuota de alimentos, declaración de unión marital de hecho, aumento y disminución de cuota de alimentos, custodia y cuidado personal, exoneración de cuota de alimentos, ofrecimiento voluntario, disolución de unión marital de hecho, regulación de visitas y gastos de embarazo y parto.

Y en materia de civil, los asuntos que se recepcionarón, en orden descendente por su frecuencia fueron: responsabilidad civil extracontractual, contrato de compraventa, contrato de mutuo, contrato de arrendamiento, responsabilidad civil contractual, reivindicatoria de dominio, cobro de sumas de dinero, responsabilidad médica y resolución de contrato de compraventa. Podemos evidenciar que durante los dos periodos la mayor frecuencia de solicitudes es en materia de familia, correspondiente al 73\% en el año 2016 y 60\% en el año 2017, del total de solicitudes recibidas, por su parte en materia civil el $27 \%$ para 2016 y el $40 \%$ para 2017, siendo los dos tipos de controversias o solicitudes presentadas por los usuarios en dicho centro.

Comparando las cifras de solicitudes de audiencias de conciliación extrajudicial en derecho atendidas en los años 2016 y 2017, con las estadísticas del Ministerio de Justicia y del Derecho (2018), se puede observar que en el año 2016, de 416 audiencias celebradas en el distrito judicial de Sincelejo, 376 fueron llevadas a cabo en el Centro de Conciliación de CECAR, siendo equivalente al 90,38\%. Asimismo, de 499 audiencias celebradas en Sincelejo para el año 2017, 337 se celebraron en nuestro Centro de Conciliación, representando el 67,54\% del total de las audiencias para ese año. Lo anterior da cuenta del gran aporte del Centro de Conciliación de CECAR a la materialización de los derechos de 
manera gratuita no solo para todo el departamento de Sucre, algunas zonas de los departamentos de Córdoba y Bolívar, sino también para los despachos judiciales, porque además de brindar a la ciudadanía un aporte a la materialización del derecho a la justicia también se está descongestionando el aparato jurisdiccional en gran medida.

\section{Usuarios atendidos en el Centro de Conciliación en los años 2016 y 2017.}

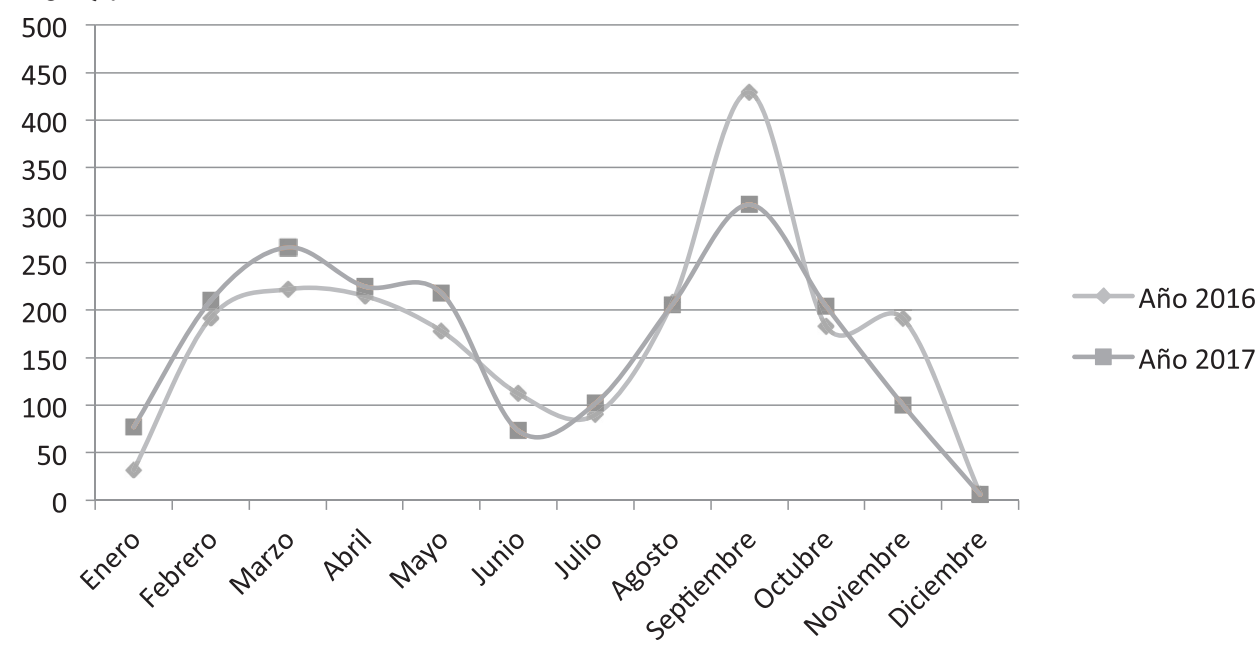

Gráfica 4. Usuarios atendidos en el Centro de Conciliación en los años 2016 y 2017.

Fuente: Elaborada por los investigadores en mención, de la información obtenida de la Coordinación del Centro de Conciliación del Consultorio Jurídico de CECAR, años 2016 y 2017.

En la gráfica 4, se estableció la afluencia de usuarios atendidos durante los años 2016 y 2017 describiéndolos numéricamente así: en el mes de enero se atendieron 32 usuarios, en el mes de febrero 210, en el mes de marzo 222, en el mes de abril 215, en mayo 178, en junio 112, en julio 102, en agosto 206, en septiembre 429, en octubre 183, en noviembre 191 y en diciembre solamente 7 usuarios, para un total de usuarios atendidos en el año 2016 de 2.087 personas. Durante ese año se realizaron 4 brigadas jurídicas en diferentes zonas del departamento de Sucre, garantizando el acceso a la administración de justicia a muchas personas de escasos recursos, en otros corregimientos y municipios del departamento.

En el año 2017 la atención de usuarios fue de 1.998 descrita numéricamente en 77 en el mes de enero, 210 en el mes de febrero, 266 en 

en derecho en el distrito judicial de Sincelejo, Colombia...

marzo, 225 en abril, 218 en mayo, 73 en junio, 102 en julio, 206 en agosto, 311 en septiembre, 204 en octubre, 100 en noviembre y 6 personas en el mes de diciembre. Los resultados anteriormente descritos, nos arrojan que el flujo de usuarios fue similar en los dos años analizados, de esta manera, encontramos que los meses de enero, junio y diciembre se presentaron pocos usuarios en razón al periodo vacacional de los estudiantes, que al parecer ya es de conocimiento a para los usuarios y optan por no solicitar los servicios durante esos meses, implicando la disminución en la recepción de solicitudes de audiencia de conciliación. Igualmente, se puede observar que en ambos años el mayor número de usuarios atendidos se dio en el mes de septiembre, fenómeno del que no comprendemos sus razones.

Por último, cabe resaltar que el Ministerio de Justicia y del Derecho, a través de la Dirección de Métodos Alternativos de Solución de Conflictos, diseñó a partir del año 2016, una jornada masiva gratuita de conciliación, denominada CONCILIATON NACIONAL. En este sentido el Centro de Conciliación de CECAR, se sumó a la jornada participando activamente en la realización de la misma, la cual fue realizada los días 24, 25 y 26 de noviembre de 2016, y los días 26, 27 y 28 de septiembre de 2017 , promoviendo así la figura de la conciliación como instrumento para resolver los conflictos entre particulares y acceder a los servicios de justicia, incitando la convivencia pacífica.

\section{Seguimientos a los acuerdos conciliatorios años 2016 y 2017}

Seguimientos año 2016

SEGUIMIENTOS A ACUERDOS

CONCILIATORIOS 2016

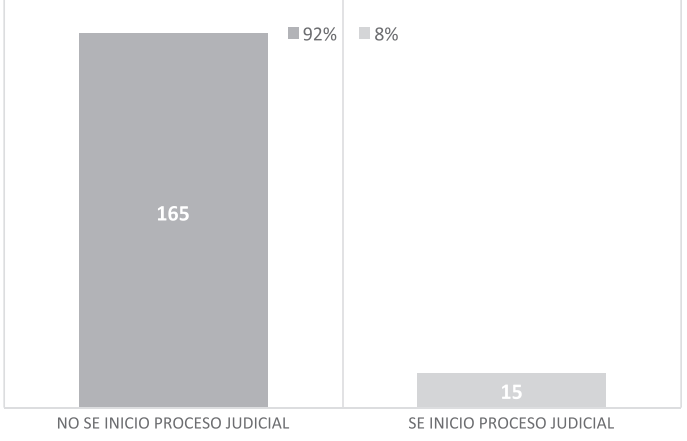

Gráfica 5. Seguimientos año 2016.

Fuente: Elaborada por los investigadores en mención, de la información obtenida de la Coordinación del Centro de Conciliación del Consultorio Jurídico de CECAR, año 2016. 
Se observa de la gráfica anterior que del total de los acuerdos conciliatorios en el año 2016, los cuales fueron 180, solo el 8\% se inició proceso judicial, en este sentido cabe resaltar la labor del Centro de Conciliación, al brindar un servicio integral, con personal idóneo, donde los acuerdo celebrados son efectivos y reales, y donde los hombres y mujeres beneficiados con ellos, no tuvieron la necesidad de acudir a otras entidades para el cumplimiento de los acuerdos en las respectivas actas de conciliación suscritas.

\section{Seguimientos año 2017.}

\section{SEGUIMIENTOS A ACUERDOS CONCILIATORIOS 2017}

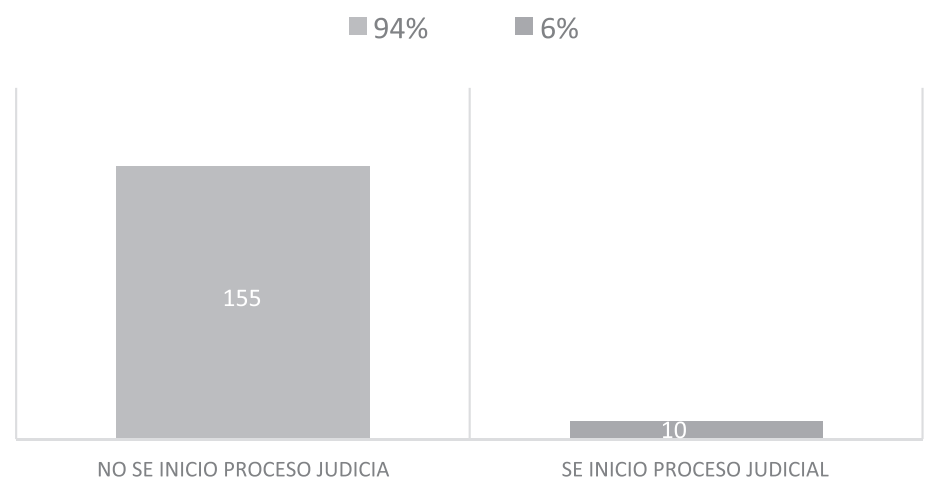

Gráfica 6. Seguimientos año 2017.

Fuente: Elaborada por los investigadores en mención, de la información obtenida de la Coordinación del Centro de Conciliación del Consultorio Jurídico de CECAR, año 2017.

Esta grafica también da cuenta, de la efectividad de los acuerdos celebrados en el Centro de Conciliación de CECAR, y del gran aporte que este Centro hace al derecho de acceso a la administración de justicia en el distrito judicial de Sincelejo, permitiendo a las personas que acuden a la búsqueda de soluciones de sus conflictos solucionarlos de manera ágil, gratuita y efectiva, prueba de ello es que en el año 2017, como lo describe el grafico, el 94\%, de los acuerdos conciliatorios no tuvo que iniciar proceso judicial.

Se puede decir que el Centro de Conciliación ha realizado muchos aportes significativos como se evidencia en las gráficas $N^{\circ} 5$ y 6 , donde los datos consignados son importantes, toda vez que un mínimo porcentaje de 
los acuerdos logrados se vieron en la necesidad de amparar sus derechos acudiendo a la justicia ordinaria.

\section{Conclusiones}

Finalmente, es preciso recalcar que el derecho de acceso a la administración de justicia se convierte en un derecho fundamental del cual deberíamos gozar a plena satisfacción todos los colombianos, en nuestro país existen diferentes mecanismos a través de los cuales podemos acceder a la administración de justicia sin necesidad de poner en movimiento el aparato jurisdiccional, dichos mecanismos son conocidos como Mecanismos Alternativos de Solución de Conflictos (MASC).

La conciliación extrajudicial en derecho es uno de los MASC más utilizados en Colombia por ser un mecanismo idóneo para acceder a la justicia de manera ágil, efectiva y en muchas ocasiones gratuita, como es el caso del servicio prestado por los Centros de Conciliación de los Consultorios Jurídicos de las Facultades de Derecho de las Universidades, los cuales tienen expresa autorización legal para administrar justicia de manera transitoria en los asuntos que por su naturaleza y cuantía le sean competentes.

Hoy en día son muchos los hombres y las mujeres que acuden a solicitar los servicios del Centro de Conciliación de CECAR, por considerar que el centro cuenta con personal idóneo e infraestructura adecuada para brindar un excelente servicio y satisfacer las necesidades de las personas atendidas, información que se puede corroborar con las gráficas anteriormente relacionadas (grafica 4), de los cuales un gran porcentaje de las audiencias realizadas terminaron de manera satisfactoria para las partes (grafica 1 y 2), convirtiéndose en un aporte significativo para el distrito judicial de Sincelejo.

Cabe resaltar que las audiencias celebradas en el Centro de Conciliación de CECAR son dirigidas por estudiantes de décimo semestre de Derecho y Ciencias Políticas, en práctica, quienes previamente han sido capacitados en MASC. Para actuar como conciliadores, los estudiantes cuentan con el apoyo de los funcionarios del Centro, lo que permite garantizar un buen servicio prestado y además refleja la calidad de la educación que brinda la Corporación Universitaria del Caribe CECAR.

Una vez analizados los resultados de la presente investigación podemos concluir que los servicios prestados por el Centro de Conciliación de CECAR, constituyen, en concordancia con el alto 
compromiso social de la Corporación, un gran aporte para la sociedad Sincelejana toda vez que es mayor el grado de satisfacción de las personas atendidas en dicho Centro, además del gran número de personas que de manera ágil, efectiva, confidencial y gratuita resolvieron sus conflictos, de forma voluntaria e integral, en los años 2016 y 2017, fortaleciendo de este modo los mecanismos alternativos de solución de conflictos que a su vez cuentan con reconocimiento constitucional como formas de administración de justicia.

Sin embargo, se deben propiciar más espacios para promover el dialogo y la autogestión de conflictos, difundiendo más los servicios del centro, sobre todo con las personas de condiciones económicas más vulnerables, quienes en un gran porcentaje aún no tienen conocimiento del mecanismo, para así contribuir más en la materialización del derecho de acceso a la justicia mediante la conciliación extrajudicial en derecho en el distrito judicial de Sincelejo en los años sucesivos, como también es necesario la implementación o certificación en calidad del Centro de Conciliación para brindar un mejor servicio, aumentar la oferta y aportar a la descongestión de los ya congestionados despachos judiciales.

\section{Referencias}

Cabanellas, G. (1976). Derecho de los Conflictos laborales. Buenos Aires: Bibliografica Omeba.

Constitución Política de Colombia, (7 de Julio de 1991).

Convención Americana Sobre Derechos Humanos, Artículo 25. ((Pacto De San José). 22 de Noviembre de 1969).

Corte Constitucional de Colombia. Sentencia C - 1083 (M.P. Jaime Araujo 24 de octubre de 2005).

Corte Constitucional de Colombia. Sentencia C-037 de 1996 (M.P. Vladimiro Naranjo Mesa: 5 de febrero de 1996).

Corte Constitucional de Colombia. Sentencia C-426 (M.P Rodrigo Escobar Gil 29 de mayo de 2002).

Corte Constitucional de Colombia. Sentencia C-893 de 2001 (M.P. Clara Inés Vargas Hernández 2001).

Corte Constitucional de Colombia. Sentencia C-985 de 2005. (M.P. Alfredo Beltrán Sierra 26 de septiembre de 2005).

Corte IDH. Opinión Consultiva OC-9/87 del 6 de octubre de 1987, Serie A No. 9, párr. 24)., Garantías judiciales en estados de emergencia (Arts. 27.2, 25 y 8 Convención Americana sobre DDHH),

De Zan, J. (2004). La etica los derechos y la Justicia. Montevideo, Uruguay: Fundacion Konrad Adenauer Stifstung E.V. 

en derecho en el distrito judicial de Sincelejo, Colombia...

Decreto 1829 , Por el cual se reglamentan algunas disposiciones de las Leyes 23 de 1991, 446 de 1998, 640de 2001 y 1563 de 2012. (27 de agosto de 2013).

Dirección de Metodos alternativos de solución de conflictos, m. d. (05 de Febrero de 2018). Concepto. Bogotá D.C., Colombia.

Escudero, M. C. (2015). Mecanismos Alternativos de Solución de Conflictos. Bogotá D.C.: Leyer.

Garcia, F. S. (2013). La Conciliación Civil y Comercial (Segunda Edición ed.). Bogotá D.C., Colombia: Ibañez S.A.S.

Hernandez, A. (2005). La Conciliación Extrajudicial en Derecho como requisito de procedibilidad. Bogotá D.C.: Leyer.

Ley 270., Estatutaria de la Administración de Justicia. (07 de marzo de 2009).

Ley 446, , Por la cual se adoptan algunas normas del Dcto 2651/91, se mod algunas del CPC, se derogan otras de la Ley 23/91 y del Decreto 2279/89, se mod y expiden normas del CCA y se dictan otras disposiciones sobre descongestión, eficiencia y acceso a la justicia (08 de julio de 1998).

López, H. (2002). Procedimiento Civil, Parte General (Octava edición ed.). Bogotá D.C., Colombia : Dupre Editores.

Mariño, P. T. (2009). El desarrollo de la Conciliación a partir de 1991. Panorama, 81 .

Ministerio de Justicia y del Derecho - Dirección de Métodos Alternativos de Solución de Conflictos y del Sistema de la Conciliación, el Arbitraje y la Amigable Composición SICAAC. (2018). Sistema de Estadísticas en Justicia. . Bogotá D.C. .

Peña, O. (2010). Conciliación Extrajudicial. Lima: Asociación Peruana de Ciencias Juridicas y Conciliación APECC.

Real Academia de la Lengua Española. (2018). Real Academia Española. Madrid. 